\title{
Effects of Dietary Aqueous Extract from Eucommia ulmoides Oliver on Growth, Muscle Composition, Amino Acid Composition and Fatty Acid Composition of Rainbow Trout (Oncorhynchus mykiss)
}

\author{
Li Yao ${ }^{1,2,3}$, AnQing Zhang ${ }^{1,2,3}$, HaiYan Zhang ${ }^{2}$, Jian Shao ${ }^{1,2,3}$, Ming Wen ${ }^{2,4}$, \\ ChangAn Wang ${ }^{5}$, HaiBo Jiang ${ }^{1,2,3, *}$ and Ming $\mathrm{Li}^{6, *}$ \\ ${ }^{1}$ Breeding and Reproduction in The Plateau Mountainous Region, Ministry of \\ Education (Guizhou University), Guiyang 550025, China \\ ${ }^{2}$ College of Animal Science, Guizhou University, Guiyang 550025, China \\ ${ }^{3}$ Special Fisheries Research Institute, Guizhou University, Guiyang 550025, China \\ ${ }^{4}$ Key Laboratory for Animal Diseases and Veterinary Public Health of Guizhou \\ Province, Guiyang 550025, China \\ ${ }^{5}$ Heilongjiang River Fisheries Research Institute, Chinese Academy of Fishery \\ Sciences, Harbin 150070, China \\ ${ }^{6}$ School of Marine Sciences, Ningbo University, Ningbo 315211, China
}

\begin{tabular}{l} 
Article Information \\
Received 19 December 2019 \\
Revised 12 Februaary 2020 \\
Accepted 04 March 2020 \\
Available online 21 February 2021 \\
Authors' Contribution \\
\hline YL, ZAQ and ZHY designed \\
the experiment and performed \\
experimental work. SJ, WM and WCA \\
analyzed the data and helped perform \\
the analysis. JHB and LM contributed \\
reagents and materials, and wrote the \\
manuscript. \\
Key words \\
Rainbow trout, Eucommia ulmoides \\
Oliver, Growth performance, Amino \\
acids, Fatty acids.
\end{tabular}

\begin{abstract}
A B S T RA C T
The study was to investigate effects of dietary Eucommia ulmoides Oliver (EUO) bark aqueous extract on growth performance and nutritional composition of rainbow trout (145.56 $\pm 4.12 \mathrm{~g}$ ) (Oncorhynchus mykiss) fed five different diets, including 0 (EUO-0), 5 (EUO-5), 10 (EUO-10), 20 (EUO-20) and 40 (EUO-40) $\mathrm{g} \mathrm{kg}^{-1}$ EUO bark aqueous extract. The muscle lipid of fish in EUO-40 was significantly higher than that in other groups ( $p<0.05)$, and fish in EUO-0 showed significantly lower muscle crude lipid content than that in EUO-5, EUO-10, and EUO-20 groups $(p<0.05)$. Fish in EUO-40 had significantly higher muscle Phe, Gly, Ala, delicious amino acids contents than that in EUO-0 $(p<0.05)$, and fish in EUO-20 had significantly higher muscle Ala content than that in EUO-0 $(p<0.05)$. Fish in EUO10 showed significantly higher $22: 1 \mathrm{n}-9$ content in muscle than that in EUO-0 $(p<0.05)$. Contents of 18:3n-6 in muscle of EUO-5 and EUO-40 groups were significantly lower than that of EUO-0 group ( $p$ $<0.05)$. Fish in EUO-40 had significantly higher 22:5n-3, 22:6n-3, and $\Sigma$ PUFA $_{\mathrm{s}} \mathrm{n}-3$ contents than that in other groups $(p<0.05)$. In conclusion, supplementation of EUO aqueous extract improved the nutritional composition of rainbow trout, and the supplemental level was estimated to be $40.00 \mathrm{~g} \mathrm{~kg}^{-1} \mathrm{diet}_{\text {. }}$
\end{abstract}

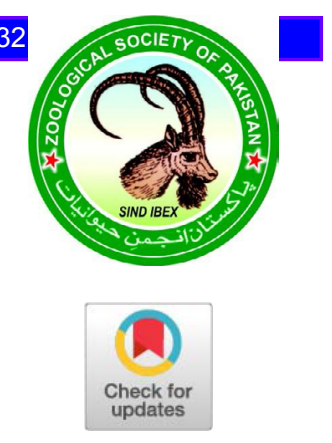

\section{INTRODUCTION}

$\mathrm{I}_{\mathrm{a}}^{\mathrm{n}}$ n developed and developing countries, fish constitutes an important food source due to its rich nutrient content. Consumers demand healthy, high-quality, natural, and fresh fish products. Rainbow trout (Oncorhynchus mykiss), as an important worldwide cold water fish species (Rezaei and Hosseini, 2008), is one of the main sources of protein, minerals, vitamins, and n-3 long-chain polyunsaturated fatty acids including eicosapentaenoic acid (EPA) and docosahexaenoic acid (DHA) (Abedi and Sahari, 2014;

\footnotetext{
* Corresponding authors: jhb99412@126.com;

liming1@nbu.edu.cn

0030-9923/2021/0002-0603 \$ 9.00/0

Copyright 2021 Zoological Society of Pakistan
}

Woynarovich et al., 2011). Its aquaculture production was more than 760,000 tones throughout the world with a total value of near 3,000,000 USD (Mirghaed et al., 2018). The quality of the products from fish is determined mainly by the nutritional value and sensory attributes of the flesh (Grigorakis, 2007); these aspects are influenced, not only among species but also within species due to several factors. Some of these factors are the age and size of the fish, the season of the year, the quality of water, the composition of diet, the feeding regime and by the stress response produced during the pre-slaughter period (Shearer, 2001; Poli, 2009; Suárez et al., 2014; BermejoPoza et al., 2017). A variety of feed additives are used to improve performance in aquaculture, to protect rainbow trout health and to improve the quantity and quality of products positively, such as plant oil (Yildiz et al., 2015), 
vitamin E (Kamireddy et al., 2011), zinc (Wu et al., 2015), selenium yeast (Wang et al., 2018), astaxanthin and canthaxanthin (Cui et al., 2009).

Eucommia ulmoides Oliver (EUO) ("Du Zhong" in Chinese, "Tu Chung" in Korean and "Tuchong" in Japanese), the sole species in the genus Eucommia and family Eucommiaceae, is a deciduous tree indigenous in China and widely cultured in eastern Asian countries ( $\mathrm{Si}$ et al., 2013). In China, it is distributed mainly in Guizhou, Hubei, Shanxi, Gansu, and Henan Provinces with a total area about 67000 hectares (Jiao et al., 2015). It is highly valued and commonly used in traditional medicines to treat various diseases, especially to relieve hypertension, protect the nervous system, treat diabetics, regulate lipid metabolism, inhibits oxidative stress (Okada et al., 1994; Geun et al., 2004; Kwon et al., 2014). The inclusion of EUO in diet improved the flesh quality of pig (Wang et al., 2007), chickens (Wang et al., 2012), grass carp, Ctenopharyngodon idella (Leng et al., 2008; Sun et al., 2017a, b), and eel, Anguilla japonica (Tanimoto et al., 1993a, b).

In China, rainbow trout aquaculture production was from 8,818 to 38,606 tones in recent ten years (MOAC, 2019). But the flesh quality of rainbow trout has been declining in recent years, showing a loose texture due to the high stocking density and the rapid growth in China. Until now, there has been no scientific report concerning the effect of EUO as a supplement in rainbow trout diet to improve flesh quality. Water is a main solvent of decocting medicinal herbs in traditional Chinese medicine. The objective of the present study was to investigate the effects of dietary aqueous extract from EUO on the growth performance and flesh quality of rainbow trout, to supply some nutritional strategies for improving the flesh quality of cultured fish.

\section{MATERIALS AND METHODS}

\section{Experimental diets and design}

During the study, the fish were fed a commercial rainbow trout feed produced by Shengsuo Fishery Feed Research Centre (Shandong, China) as the basal diet. The proximate composition was as follows: crude protein 420 $\mathrm{g} \mathrm{kg}^{-1}$, lipid $180 \mathrm{~g} \mathrm{~kg}^{-1}$, crude fibre $60 \mathrm{~g} \mathrm{~kg}^{-1}$, and crude ash $80 \mathrm{~g} \mathrm{~kg}^{-1}$. The diets were prepared to contain $0 \mathrm{~g}$ $\mathrm{kg}^{-1}$ (control, EUO-0), $5.00 \mathrm{~g} \mathrm{~kg}^{-1}$ (EUO-5), $10.00 \mathrm{~g} \mathrm{~kg}^{-1}$ (EUO-10), $20.00 \mathrm{~g} \mathrm{~kg}^{-1}$ (EUO-20) and $40.00 \mathrm{~g} \mathrm{~kg}^{-1}$ (EUO40) Eucommia ulmoides Oliver barks extract (EUO). The main nutritional component and active substance content of Eucommia ulmoides Oliver bark aqueous extract is in Table I. Commercial rainbow trout feed was ground through 40-mesh size and then was weighed. EUO was purchased from a local hospital, which was cut and dried at room temperature for 3 days. After dried, the EUO (0 g, $5.0 \mathrm{~g}, 10.0 \mathrm{~g}, 20.0 \mathrm{~g}$ or $40.0 \mathrm{~g}$, respectively) was lightly boiled in $1000 \mathrm{ml}$ of distilled water for $4 \mathrm{~h}$, concentrated to $100 \mathrm{ml}$, and then aqueous extract from EUO $(10 \%, \mathrm{v} / \mathrm{w})$ and water $(30 \%, \mathrm{v} / \mathrm{w})$ were added to feed form a soft dough, and then were mixed to facilitate pelleting by a granulator (330; Ou-siqi Mechanical and Electrical Equipment Co., Ltd, Zhejiang, China). The pellets (1.0 mm diameter) were extruded and air-dried and stored at $-20^{\circ} \mathrm{C}$ until use.

\section{Fish and sampling}

The feeding activities were performed for $70 \mathrm{~d}$ at a private rainbow trout farm (Dashan rainbow trout farm, Dafang, Guizhou, China). A total of 450 rainbow trout with an initial body weight of $145.56 \pm 4.12 \mathrm{~g}$ were randomly allocated in 15 cages $(1 \mathrm{~m} \times 1 \mathrm{~m} \times 1.2 \mathrm{~m})$ with a water depth of $0.8 \mathrm{~m}$ and 30 fish per cage. The cages were located in three outdoor cement pools $(18 \mathrm{~m} \times 3.8 \mathrm{~m} \times 1.2 \mathrm{~m})$ with each pool containing five cages from five treatments. During the feeding period, the fish were fed manually to apparent satiation four times per day $(08: 00,12: 00,15: 00$, 18: 00) with a daily feeding rate of $2.0 \%-4.0 \%$ of body weight. Fish in each cage were weighed every 2 weeks for feed adjustments throughout the experimental period. Dissolved oxygen, temperature, $\mathrm{pH}$, ammonia nitrogen, and velocity of water were $>7.5 \mathrm{mg} \mathrm{L}^{-1}, 12.8-14.1{ }^{\circ} \mathrm{C}$, $7.6-8.4,<0.05 \mathrm{mg} \mathrm{L}^{-1}$, and $0.04 \mathrm{~m} \mathrm{~s}^{-1}$.

Table I.- Main nutritional component and active substance content of Eucommia ulmoides Oliver bark aqueous extract.

\begin{tabular}{|c|c|c|c|c|c|}
\hline Treatment & EUO-0 & EUO-5 & EUO-10 & EUO-20 & EUO-40 \\
\hline Crude protein (g 100 $\mathrm{ml}^{-1}$ aqueous extract) & 0 & $0.10 \pm 0.0030$ & $0.28 \pm 0.0040$ & $0.30 \pm 0.0050$ & $0.37 \pm 0.050$ \\
\hline Crude lipid (g 100 $\mathrm{ml}^{-1}$ aqueous extract) & 0 & $0.33 \pm 0.028$ & $0.48 \pm 0.034$ & $0.50 \pm 0.042$ & $0.85 \pm 0.040$ \\
\hline Ash (g $100 \mathrm{ml}^{-1}$ aqueous extract) & 0 & $0.12 \pm 0.0089$ & $0.15 \pm 0.0050$ & $0.25 \pm 0.0035$ & $0.51 \pm 0.0071$ \\
\hline Chlorogenic acid (\%) & 0 & 0.17 & 0.20 & 0.15 & 0.15 \\
\hline Aucubin $(\%)$ & 0 & 1.64 & 1.90 & 1.29 & 0.87 \\
\hline Geniposide (\%) & 0 & 0.38 & 0.45 & 0.24 & 0.22 \\
\hline Geniposidic acid (\%) & 0 & 1.45 & 1.59 & 1.18 & 0.96 \\
\hline
\end{tabular}


All fish in each cage were weighed and counted at the beginning and at the end of the experiment. Dead fish were removed and recorded daily. At the end, twelve fish from each cage were randomly collected and were anesthetized with $200 \mathrm{mg} \mathrm{L}^{-1} \mathrm{MS}-222$. Six fish were used for the analysis of muscle composition, and six fish were used to obtain the weights of whole body, viscera and liver. All samples were stored at $-80^{\circ} \mathrm{C}$ before analysis.

\section{Growth performance and husbandry parameters}

Weight gain rate (WGR), specific growth rate (SGR), condition factor (CF) viscerosomatic index (VSI), hepatosomatic index (HSI), survival rate (SR) were calculated as follows:

$$
\begin{gathered}
\text { WGR }(\%)=100 \times \frac{\text { Final body wt }- \text { Initial body wt }}{\text { Initial body wt }} \\
\text { SGR }\left(\% \text { day }^{-1}\right)=100 \times \frac{\ln (\text { Final wt }- \text { Initial wt })}{\text { Days of the experiment }} \\
\text { HSI }(\%)=100 \times \frac{\text { Final liver wt }(\mathrm{g})}{\text { Final body wt }(\mathrm{g})} \\
\text { VSI }(\%)=100 \times \frac{\text { Final viscera wt }(\mathrm{g})}{\text { Final body wt }(\mathrm{g})} \\
\text { CF }\left(\mathrm{g} / \mathrm{cm}^{3}\right)=100 \times \frac{\text { Final body wt }(\mathrm{g})}{\text { Body length }\left(\mathrm{cm}^{3}\right)} \\
\text { Survival }(\%)=100 \times \frac{\text { Final fish number }}{\text { Initial fish number }}
\end{gathered}
$$

\section{Muscle and diets proximate composition}

Chemical compositions of diets and muscle including lipid, protein, moisture and ash were analyzed according to standard methods (AOAC, 2005). All analyses were performed in five replicates.
Muscle amino acids and fatty acids

The amino acid compositions of ingredients were determined in triplicate with an automatic amino acid analyzer (LKB Biochrom Ltd., Cambridge, UK). Total lipid of muscle samples were extracted using chloroform: methanol $(2: 1, \mathrm{v} / \mathrm{v})$ according to the method of Folch et al. (1957). The saponifiable lipids were converted to methyl esters by using the standard boron tri-fluoridemethanol method (Morrison and Smith, 1964). Fatty acids methyl esters (FAME) were analysed on an Agilent 6890 gas chromatograph (Agilent Technologies, Santa Clara, CA, USA), equipped with a flame ionization detector (FID) and a SP-2560 fused silica capillary column (100 $\mathrm{m}$ long, $0.25 \mathrm{~mm}$ i.d. and $0.20 \mu \mathrm{m}$ thick). Injector and detector temperatures were 270 and $280^{\circ} \mathrm{C}$, respectively. Column temperature was held at $120^{\circ} \mathrm{C}$ for $5 \mathrm{~min}$ then programmed to increase at $3^{\circ} \mathrm{C} \mathrm{min}^{-1}$ up to $240^{\circ} \mathrm{C}$, where it was maintained for $20 \mathrm{~min}$. Carrier gas was helium $\left(2 \mathrm{~mL} \mathrm{~min}^{-1}\right.$ ), and the split ratio was 30:1. Identification of fatty acids was carried out by comparing the sample FAME peak relative retention times with Sigma-Aldrich (St. Louis, MO, USA) standards. The individual FAME was identified by comparing the retention times of the authentic standard mixtures. The amount of each fatty acid was calculated using the peak area normalization method as a relative per cent value to the total fatty acids.

\section{Statistical analysis}

Data were expressed as means \pm standard deviation (SD). All statistical evaluations were analyzed using oneway analysis of variance (ANOVA) by the software SPSS 11.0 (SPSS Inc., Chicago, IL, USA) for Windows. Prior to the statistical tests, data were examined for normality and homogeneity of variances. Differences between the means were tested by Duncan's multiple-range tests. The level of significance was set at $p<0.05$.

Table II.- Effects of Eucommia ulmoides Oliver bark aqueous extract on growth performance of rainbow trout.

\begin{tabular}{lccccc}
\hline Parameters & EUO-0 & EUO-5 & EUO-10 & EUO-20 & EUO-40 \\
\hline IBW (g) & $144.44 \pm 5.09$ & $144.45 \pm 3.85$ & $145.55 \pm 3.85$ & $145.56 \pm 5.09$ & $147.78 \pm 5.09$ \\
FBW (g) & $380.08 \pm 23.47$ & $393.14 \pm 9.14$ & $394.84 \pm 22.30$ & $386.59 \pm 18.04$ & $386.51 \pm 6.67$ \\
WGR (\%) & $163.46 \pm 20.84$ & $172.19 \pm 1.54$ & $171.15 \pm 9.48$ & $165.71 \pm 12.39$ & $161.82 \pm 12.13$ \\
SGR (\% day $\left.{ }^{-1}\right)$ & $1.38 \pm 0.11$ & $1.43 \pm 0.081$ & $1.42 \pm 0.050$ & $1.40 \pm 0.067$ & $1.37 \pm 0.067$ \\
HSI (\%) & $0.86 \pm 0.027$ & $0.86 \pm 0.016$ & $0.84 \pm 0.070$ & $0.95 \pm 0.023$ & $0.86 \pm 0.037$ \\
VSI (\%) & $10.63 \pm 0.88$ & $10.90 \pm 0.88$ & $11.33 \pm 0.45$ & $11.21 \pm 0.78$ & $11.09 \pm 0.89$ \\
CF (g/cm $\left.{ }^{3}\right)$ & $1.47 \pm 0.029$ & $1.43 \pm 0.021$ & $1.45 \pm 0.029$ & $1.45 \pm 0.028$ & $1.44 \pm 0.032$ \\
SR/\% & $98.89 \pm 1.92$ & $98.89 \pm 1.92$ & $97.78 \pm 3.85$ & $96.67 \pm 3.33$ & $98.89 \pm 1.92$ \\
\hline
\end{tabular}

Values in the same row with different superscripts indicate significant differences $(\mathrm{P}<0.05)$. IBW, initial body weight; $\mathrm{CF}$, condition factor; FBW, final body weight; HSI, hepatosomatic index; SGR, specific growth rate; VSI, viscerosomatic index; SR: survival rate; WGR, weight gain rate. 


\section{RESULTS}

Growth performance

Results of growth performance are presented in Table II. FBW, WG, SGR, HSI, VSI, CF and SR show no significant difference among all treatment $(p>0.05)$.

\section{Muscle composition}

Muscle composition ( $\mathrm{g} \mathrm{kg}^{-1}$, wet basis) of the rainbow trout fed with different aqueous extract from EUO levels is presented in Table III. The fish fed EUO-40 diet showed significantly higher muscle crude lipid content than the fish fed other diets $(p<0.05)$, and the fish fed EUO-5, EUO-
10, or EUO-20 diet showed significantly higher muscle crude lipid content than the EUO-0 group $(p<0.05)$. For moisture, crude protein and crude ash, there were no significant differences among all treatments $(p>0.05)$.

Muscle amino acid and fatty acid composition

The effects of the dietary aqueous extract from EUO on the amino acid profile in the muscle of rainbow trout are shown in Table IV. These results indicated that most of the amino acid concentrations in muscle were not significantly affected by dietary EUO levels compared with control group (EUO-0) $(p>0.05)$, except for Val, Phe, Gly, Ala and DAA $(p<0.05)$. Fish fed EUO-5 diet had lower Val

Table III.- Effects of Eucommia ulmoides Oliver bark aqueous extract on proximate composition in muscle of rainbow trout ( $\mathrm{g} \mathrm{kg}^{-1}$, wet weight).

\begin{tabular}{lccccc}
\hline Treatment & EUO-0 & EUO-5 & EUO-10 & EUO-20 & EUO-40 \\
\hline Moisture & $753.26 \pm 0.44$ & $751.24 \pm 0.35$ & $752.08 \pm 0.44$ & $752.09 \pm 0.36$ & $751.13 \pm 0.21$ \\
Crude protein & $199.26 \pm 1.36$ & $199.34 \pm 1.37$ & $200.60 \pm 1.07$ & $199.11 \pm 1.27$ & $200.90 \pm 0.53$ \\
Crude lipid & $29.96 \pm 0.37^{\mathrm{a}}$ & $33.56 \pm 0.24^{\mathrm{b}}$ & $33.40 \pm 0.82^{\mathrm{b}}$ & $33.56 \pm 0.25^{\mathrm{b}}$ & $34.71 \pm 0.54^{\mathrm{c}}$ \\
Crude ash & $12.58 \pm 0.093$ & $12.71 \pm 0.030$ & $12.88 \pm 0.025$ & $12.89 \pm 0.032$ & $12.58 \pm 0.17$ \\
\hline
\end{tabular}

Values in the same row with different superscripts indicate significant differences $(P<0.05)$.

Table IV.- Effects of Eucommia ulmoides Oliver bark aqueous extract on muscle amino acid composition of rainbow

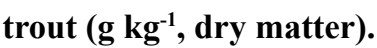

\begin{tabular}{lccccc}
\hline Amino acids & EUO-0 & EUO-5 & EUO-10 & EUO-20 & EUO-40 \\
\hline EAA & & & & & \\
Thr & $35.40 \pm 0.79$ & $35.37 \pm 0.25$ & $36.00 \pm 0.26$ & $35.27 \pm 0.71$ & $35.87 \pm 0.60$ \\
Val & $38.63 \pm 0.47^{\mathrm{b}}$ & $37.60 \pm 0.30^{\mathrm{a}}$ & $38.90 \pm 0.52^{\mathrm{b}}$ & $38.80 \pm 0.46^{\mathrm{b}}$ & $39.03 \pm 0.85^{\mathrm{b}}$ \\
Met & $21.57 \pm 0.83$ & $21.43 \pm 0.96$ & $19.20 \pm 0.92$ & $21.60 \pm 0.71$ & $20.97 \pm 0.83$ \\
Ile & $37.23 \pm 0.86$ & $36.67 \pm 0.31$ & $37.77 \pm 0.40$ & $37.83 \pm 0.35$ & $37.77 \pm 0.64$ \\
Leu & $62.67 \pm 0.57^{\mathrm{ab}}$ & $61.87 \pm 0.49^{\mathrm{a}}$ & $63.53 \pm 0.49^{\mathrm{b}}$ & $63.20 \pm 0.76^{\mathrm{ab}}$ & $63.33 \pm 0.95^{\mathrm{b}}$ \\
Phe & $33.37 \pm 0.35^{\mathrm{a}}$ & $33.37 \pm 0.25^{\mathrm{a}}$ & $33.60 \pm 0.36^{\mathrm{ab}}$ & $33.83 \pm 0.29^{\mathrm{ab}}$ & $34.20 \pm 0.66^{\mathrm{b}}$ \\
His & $17.87 \pm 0.60^{\mathrm{ab}}$ & $17.70 \pm 0.17^{\mathrm{a}}$ & $18.47 \pm 0.25^{\mathrm{b}}$ & $18.00 \pm 0.20^{\mathrm{ab}}$ & $18.23 \pm 0.12^{\mathrm{ab}}$ \\
Lys & $73.13 \pm 0.80$ & $72.90 \pm 0.20$ & $74.57 \pm 0.17$ & $73.37 \pm 0.78$ & $74.37 \pm 0.51$ \\
Arg & $48.67 \pm 0.41$ & $47.40 \pm 0.69$ & $49.13 \pm 0.13$ & $47.40 \pm 0.11$ & $48.57 \pm 0.37$ \\
TEAA & $368.53 \pm 4.97$ & $364.30 \pm 4.19$ & $371.17 \pm 6.30$ & $369.30 \pm 6.21$ & $372.33 \pm 6.31$ \\
NEAA & & & & $76.57 \pm 0.84^{\mathrm{a}}$ & $78.20 \pm 0.85^{\mathrm{b}}$ \\
Asp & $76.93 \pm 1.21^{\mathrm{ab}}$ & $76.63 \pm 0.55^{\mathrm{a}}$ & $78.33 \pm 0.21^{\mathrm{b}}$ & $110.10 \pm 1.87$ & $113.13 \pm 1.60$ \\
Glu & $111.33 \pm 2.25$ & $111.83 \pm 1.61$ & $112.23 \pm 0.47$ & $39.97 \pm 0.15^{\mathrm{a}}$ & $42.13 \pm 1.79^{\mathrm{b}}$ \\
Gly & $39.67 \pm 0.21^{\mathrm{a}}$ & $40.53 \pm 1.36^{\mathrm{ab}}$ & $39.80 \pm 1.08^{\mathrm{a}}$ & $48.87 \pm 0.35^{\mathrm{c}}$ & $49.73 \pm 0.35^{\mathrm{d}}$ \\
Ala & $48.30 \pm 0.17^{\mathrm{b}}$ & $47.37 \pm 0.40^{\mathrm{a}}$ & $48.37 \pm 0.058^{\mathrm{bc}}$ & $30.33 \pm 0.57^{\mathrm{a}}$ & $31.40 \pm 0.17^{\mathrm{b}}$ \\
Ser & $30.87 \pm 0.55^{\mathrm{ab}}$ & $31.27 \pm 0.50^{\mathrm{b}}$ & $31.50 \pm 0.20^{\mathrm{b}}$ & $29.50 \pm 0.44$ & $29.70 \pm 0.87$ \\
Tyr & $29.70 \pm 0.61$ & $29.60 \pm 0.55$ & $30.17 \pm 0.21$ & $28.57 \pm 0.12$ & $28.90 \pm 0.40$ \\
Pro & $29.13 \pm 0.73$ & $27.53 \pm 0.94$ & $31.90 \pm 0.95$ & $363.90 \pm 3.80$ & $373.20 \pm 3.40$ \\
TNEAA & $365.93 \pm 8.05$ & $364.77 \pm 3.00$ & $372.30 \pm 3.84$ & $275.50 \pm 2.21^{\mathrm{a}}$ & $283.20 \pm 3.53^{\mathrm{b}}$ \\
DAA & $276.23 \pm 3.27^{\mathrm{a}}$ & $276.37 \pm 2.30^{\mathrm{a}}$ & $278.73 \pm 1.59^{\mathrm{ab}}$ & $733.20 \pm 9.79$ & $745.53 \pm 8.59$ \\
TAA & $734.47 \pm 6.72$ & $729.07 \pm 6.19$ & $743.47 \pm 6.31$ & & \\
\hline
\end{tabular}

Values in the same row with different superscripts alphabets indicate significant differences $(P<0.05)(\mathrm{n}=3)$. EAA, essential amino acids; NEAA, nonessential amino acids; TEAA, total essential amino acids; TNEAA, total non-essential amino acids; DAA (Asp, Gly, Glu, Ala), delicious amino acids; TAA, total amino acids. 
Table V.- Effects of Eucommia ulmoides Oliver bark aqueous extract on muscle fatty acid composition of rainbow trout ( $\mathrm{g} \mathrm{kg}^{-1}$, dry matter).

\begin{tabular}{|c|c|c|c|c|c|}
\hline Fatty acids & EUO-0 & EUO-5 & EUO-10 & EUO-20 & EUO-40 \\
\hline $\mathrm{C} 8: 0$ & ND & ND & ND & ND & ND \\
\hline $\mathrm{C} 10: 0$ & ND & ND & ND & ND & ND \\
\hline C11:0 & ND & ND & ND & ND & ND \\
\hline $\mathrm{C} 12: 0$ & $0.55 \pm 0.050$ & $0.55 \pm 0.042$ & $0.56 \pm 0.021$ & $0.55 \pm 0.058$ & $0.54 \pm 0.010$ \\
\hline C13:0 & ND & ND & ND & ND & ND \\
\hline C14:0 & $47.87 \pm 1.20$ & $48.32 \pm 0.42$ & $48.82 \pm 0.53$ & $48.75 \pm 1.01$ & $48.72 \pm 1.87$ \\
\hline C15:0 & $5.34 \pm 0.078$ & $5.23 \pm 0.028$ & $5.21 \pm 0.13$ & $5.09 \pm 0.045$ & $5.13 \pm 0.058$ \\
\hline C16:0 & $207.68 \pm 0.60$ & $206.28 \pm 4.40$ & $210.87 \pm 5.43$ & $207.46 \pm 4.46$ & $209.16 \pm 1.17$ \\
\hline C17:0 & $3.17 \pm 0.076$ & $3.17 \pm 0.068$ & $3.10 \pm 0.045$ & $3.05 \pm 0.080$ & $3.11 \pm 0.012$ \\
\hline C18:0 & $45.26 \pm 0.74^{\mathrm{ab}}$ & $44.46 \pm 0.69^{\mathrm{a}}$ & $46.63 \pm 0.12^{b}$ & $45.29 \pm 0.56^{\mathrm{ab}}$ & $45.10 \pm 0.41^{\mathrm{ab}}$ \\
\hline $\mathrm{C} 20: 0$ & $0.76 \pm 0.014$ & $0.86 \pm 0.046$ & $0.81 \pm 0.064$ & $0.81 \pm 0.060$ & $0.82 \pm 0.012$ \\
\hline $\mathrm{C} 21: 0$ & ND & ND & ND & ND & ND \\
\hline $\mathrm{C} 20: 2$ & $0.92 \pm 0.020$ & $0.99 \pm 0.017$ & $0.91 \pm 0.026$ & $0.96 \pm 0.042$ & $0.97 \pm 0.064$ \\
\hline $\mathrm{C} 22: 0$ & $1.44 \pm 0.051^{\mathrm{ab}}$ & $1.42 \pm 0.087^{\mathrm{ab}}$ & $1.48 \pm 0.025^{\mathrm{b}}$ & $1.37 \pm 0.047^{\mathrm{a}}$ & $1.44 \pm 0.032^{\mathrm{ab}}$ \\
\hline $\mathrm{C} 23: 0$ & ND & ND & ND & ND & ND \\
\hline $\mathrm{C} 24: 0$ & ND & ND & ND & ND & ND \\
\hline C14:1 & $0.41 \pm 0.025$ & $0.41 \pm 0.032$ & $0.42 \pm 0.0058$ & $0.44 \pm 0.012$ & $0.40 \pm 0.015$ \\
\hline C15:1 & ND & ND & ND & ND & ND \\
\hline C16:1 & $68.35 \pm 2.13$ & $69.06 \pm 3.42$ & $68.86 \pm 2.24$ & $70.57 \pm 1.98$ & $67.96 \pm 3.06$ \\
\hline $\mathrm{C} 17: 1$ & $6.41 \pm 0.088$ & $5.93 \pm 0.022$ & $5.83 \pm 0.055$ & $6.05 \pm 0.027$ & $5.95 \pm 0.042$ \\
\hline C18:1- trans(n-9) & $1.11 \pm 0.026$ & $1.08 \pm 0.012$ & $1.16 \pm 0.049$ & $1.28 \pm 0.018$ & $1.12 \pm 0.032$ \\
\hline C18:1-cis (n-9) & $230.23 \pm 0.79$ & $229.66 \pm 3.25$ & $230.95 \pm 0.65$ & $232.20 \pm 3.60$ & $225.77 \pm 1.67$ \\
\hline C20:1n-9 & $5.71 \pm 0.15$ & $6.07 \pm 0.054$ & $5.95 \pm 0.12$ & $6.00 \pm 0.037$ & $6.27 \pm 0.12$ \\
\hline $\mathrm{C} 22: 1 \mathrm{n}-9$ & $1.14 \pm 0.036^{\mathrm{a}}$ & $1.14 \pm 0.012^{\mathrm{a}}$ & $1.39 \pm 0.011^{\mathrm{b}}$ & $1.31 \pm 0.034^{\mathrm{ab}}$ & $1.29 \pm 0.015^{\mathrm{ab}}$ \\
\hline $\mathrm{C} 24: 1 \mathrm{n}-9$ & $2.39 \pm 0.024$ & $2.43 \pm 0.015$ & $2.46 \pm 0.013$ & $2.44 \pm 0.071$ & $2.53 \pm 0.067$ \\
\hline C18:2-trans(n-6) & $0.82 \pm 0.018$ & $0.83 \pm 0.018$ & $0.86 \pm 0.027$ & $0.73 \pm 0.056$ & $1.27 \pm 0.016$ \\
\hline C18:2-cis(n-6) & $163.83 \pm 1.99$ & $162.79 \pm 4.13$ & $154.84 \pm 3.52$ & $157.19 \pm 3.54$ & $154.43 \pm 4.79$ \\
\hline$C 18: 3 n-3$ & $20.49 \pm 0.57$ & $20.47 \pm 0.21$ & $19.23 \pm 0.29$ & $19.64 \pm 0.67$ & $19.48 \pm 0.33$ \\
\hline C18:3n-6 & $4.09 \pm 0.045^{\mathrm{b}}$ & $3.16 \pm 0.015^{\mathrm{a}}$ & $3.59 \pm 0.079^{\mathrm{ab}}$ & $3.43 \pm 0.053^{\mathrm{ab}}$ & $2.92 \pm 0.068^{\mathrm{a}}$ \\
\hline$C 20: 3 n-3$ & $1.46 \pm 0.061$ & $1.52 \pm 0.014$ & $1.55 \pm 0.040$ & $1.46 \pm 0.042$ & $1.42 \pm 0.049$ \\
\hline$C 20: 3 n-6$ & $11.30 \pm 0.26$ & $10.89 \pm 0.19$ & $10.84 \pm 0.30$ & $10.88 \pm 0.25$ & $10.82 \pm 0.18$ \\
\hline$C 20: 4 n-6$ & $6.97 \pm 0.035$ & $6.91 \pm 0.075$ & $7.18 \pm 0.036$ & $6.88 \pm 0.064$ & $7.26 \pm 0.026$ \\
\hline$C 20: 5 n-3$ & $54.53 \pm 1.47$ & $56.12 \pm 1.21$ & $54.92 \pm 1.04$ & $55.40 \pm 1.34$ & $57.08 \pm 1.11$ \\
\hline $\mathrm{C} 22: 2$ & $4.92 \pm 0.014$ & $5.37 \pm 0.042$ & $5.10 \pm 0.018$ & $4.98 \pm 0.039$ & $5.22 \pm 0.22$ \\
\hline$C 22: 5 n-3$ & $13.62 \pm 0.20^{\mathrm{a}}$ & $14.09 \pm 0.097^{\mathrm{a}}$ & $13.84 \pm 0.056^{\mathrm{a}}$ & $14.06 \pm 0.37^{\mathrm{a}}$ & $15.51 \pm 0.090^{b}$ \\
\hline$C 22: 6 n-3$ & $89.21 \pm 2.57^{\mathrm{a}}$ & $90.79 \pm 3.66^{\mathrm{a}}$ & $92.64 \pm 2.33^{\mathrm{a}}$ & $91.72 \pm 3.06^{\mathrm{a}}$ & $98.30 \pm 3.18^{b}$ \\
\hline$\Sigma \mathrm{SFA}_{\mathrm{S}}{ }^{\mathrm{a}}$ & $312.08 \pm 1.32$ & $310.28 \pm 4.63$ & $317.49 \pm 5.34$ & $312.05 \pm 6.68$ & $314.02 \pm 2.98$ \\
\hline$\Sigma \mathrm{UFA}_{\mathrm{S}}^{\mathrm{b}}$ & $687.92 \pm 0.31$ & $689.72 \pm 4.63$ & $682.52 \pm 5.34$ & $687.95 \pm 6.69$ & $685.98 \pm 2.98$ \\
\hline$\Sigma$ MUFA $_{\mathrm{S}}{ }^{\mathrm{c}}$ & $315.75 \pm 0.98$ & $315.79 \pm 7.27$ & $317.01 \pm 7.05$ & $320.30 \pm 5.31$ & $311.29 \pm 5.70$ \\
\hline$\Sigma$ PUFA $_{\mathrm{s}}^{\mathrm{d}}$ & $372.17 \pm 1.25$ & $373.93 \pm 6.64$ & $365.51 \pm 4.55$ & $367.65 \pm 4.67$ & $374.69 \pm 8.53$ \\
\hline$\Sigma$ PUFA $_{\mathrm{S}} n-3^{\mathrm{e}}$ & $179.31 \pm 1.50^{\mathrm{a}}$ & $183.00 \pm 5.12^{\mathrm{a}}$ & $182.19 \pm 2.81^{\mathrm{a}}$ & $182.27 \pm 3.47^{\mathrm{a}}$ & $191.79 \pm 3.53^{b}$ \\
\hline$\Sigma$ PUFA $_{S} n-6^{f}$ & $188.01 \pm 4.06$ & $184.58 \pm 3.38$ & $177.30 \pm 3.77$ & $179.43 \pm 2.80$ & $176.71 \pm 5.25$ \\
\hline$n-3 / n-6$ & $0.95 \pm 0.027$ & $0.99 \pm 0.078$ & $1.03 \pm 0.028$ & $1.02 \pm 0.055$ & $1.09 \pm 0.015$ \\
\hline $\mathrm{IA}^{\mathrm{g}}$ & $0.59 \pm 0.0087$ & $0.59 \pm 0.0081$ & $0.60 \pm 0.013$ & $0.59 \pm 0.016$ & $0.60 \pm 0.015$ \\
\hline $\mathrm{IT}^{\mathrm{h}}$ & $0.33 \pm 0.00090$ & $0.33 \pm 0.0099$ & $0.34 \pm 0.0056$ & $0.33 \pm 0.0059$ & $0.32 \pm 0.0064$ \\
\hline
\end{tabular}

${ }^{a}$ Staturated fatty acid; ${ }^{b}$ Unstaturated fatty acid; ${ }^{c}$ Monounstaturated fatty acid; ${ }^{d}$ Polyunstaturated fatty acid; ${ }^{e} \mathrm{n}-3$ polyunsaturated fatty acids; ${ }^{\mathrm{f}} \mathrm{n}-3$ polyunsaturated fatty acids; 'Index of atherogenicity $=(12: 0+4 \times 14: 0+16: 0) /[(\mathrm{n}-6+\mathrm{n}-3)$ PUFA $+18: 1+$ the sum of other MUFA] (Suárez et al., 2014); ${ }^{\text {IIndex of thrombogenicity }=(14: 0+16: 0+18: 0) /\left[0.5 \times 18: 1+0.5 \times \Sigma \text { MUFA }_{\mathrm{s}}+0.5 \times \Sigma \text { PUFA }_{\mathrm{s}} \mathrm{n}-6+3 \times \Sigma \text { PUFA }_{\mathrm{s}} \mathrm{n}-3+(\mathrm{n}-3 / \mathrm{n}-6)\right](\text { Suárez et }}$ al., 2014). ND, not detected. Values in the same row with different superscripts indicate significant differences $(P<0.05)(\mathrm{n}=3)$. 
than those fed the other diets $(p<0.05)$. Fish fed EUO-0 and EUO-5 diets had lower Phe than that fed EUO-40 diet $(p<0.05)$. Fish fed EUO-0, EUO-10 and EUO-20 diets had lower Gly than that fed EUO-40 diet $(P<0.05)$. Fish fed EUO-40 diet had the highest Ala among all groups $(p<$ 0.05 ). And fish fed EUO-0 diet had lower Ala than that fed EUO-20 and EUO-40 diets $(p<0.05)$, but had higher Ala than that fed EUO-5 diet $(p<0.05)$. Fish fed EUO-40 diets had higher DAA than that fed EUO-0, EUO-5 and EUO20 diets $(p<0.05)$. However, TEAA, TNEAA, TAA levels in muscle were not significantly influenced by the dietary different aqueous extract from EUO levels $(p>0.05)$.

As shown in Table V, the levels of most fatty acids were similar in muscle of fish fed different diet. The fatty acids in fish showed that the most abundant component was 18:1-cis (n-9) with values from 225.77 to $232.20 \mathrm{~g}$ $\mathrm{kg}^{-1}$, but there were also rich in 16:0, 18:2-cis (n-6) with an interval from 206.28 to $210.87 \mathrm{~g} \mathrm{~kg}^{-1}, 154.43$ to 163.83 $\mathrm{g} \mathrm{kg}^{-1}$, respectively. 22:1n-9 in muscle of EUO-0, EUO-5 group was lower than that of EUO-10 group $(p<0.05)$. 18:3n-6 in muscle of EUO-0 group was higher than that of EUO-5 and EUO-40 groups ( $p<0.05)$. 22:5n-3, 22:6n-3, $\Sigma$ PUFAs n-3 in muscle of EUO-40 group was significantly higher than those in muscle of other groups $(p<0.05)$, but there was no significant difference on 22:5n-3, 22:6n-3, $\Sigma$ PUFAs n-3 in muscle among EUO-0, EUO-5, EUO-10 and EUO-20 groups $(p>0.05)$.

\section{DISCUSSION}

The current study showed no improvement in growth performance by dietary aqueous extract of EUO. Similarly, Sun et al. (2018) found that dietary $20.00 \mathrm{~g} \mathrm{~kg}^{-1}$ aqueous extract (water-soluble) and residue (water-insoluble) of EUO could not cause positive effect of the growth in Ctenopharyngodon idella (initial body weight, $47.1 \pm 0.8$ g). However, some studies showed the positive effects of dietary EUO on the growth performance of culture animals. Another study by Sun et al. (2017b) indicated that WG of $C$. idella (initial body weight, $95.2 \pm 0.6 \mathrm{~g}$ ) was significantly increased by $20.00 \mathrm{~g} \mathrm{~kg}^{-1}$ aqueous extract (water-soluble) and residue (water-insoluble) of EUO. The inconsistent results of $C$. idella were probably due to the differences in body size, producing area, processing method, storing time and active compound content in EUO. And dietary 20.00 and $30.00 \mathrm{~g} \mathrm{~kg}^{-1}$ aqueous extract (water-soluble) and residue (water-insoluble) of EUO significantly increased the WG of Litopenaeus vannamei (Liu, 2013). In the study by Wang et al. (2007), $1.5 \mathrm{~g}$ $\mathrm{kg}^{-1}$ dietary EUO promoted the daily weight gain in pig. Meng et al. (2007) found that $1.5 \mathrm{~g} \mathrm{~kg}^{-1} \mathrm{EUO}$ powder significantly increased WG in C. idella (initial body weight, $37.0 \pm 3.0 \mathrm{~g}$ ). The supplementation of $40.0 \mathrm{~g} \mathrm{~kg}^{-1}$
EUO leaf powder significantly increased WG in C. idella (initial body weight, $425.8 \pm 37.6 \mathrm{~g}$ ) (Leng et al., 2008), and $1.5 \mathrm{~g} \mathrm{~kg}^{-1}$ EUO leaf extract significantly increased SGR in Carassius autatus gibelio (Shi et al., 2008). The inconsistent reports of different species were probably ascribed to the species, body size, diet composition, environment, feeding period, active compounds contents of EUO from different parts and locations, etc. Zhou et al. (2009) reported that there are significant differences in the components and their contents of the relevant essential oil and flavones from EUO barks and leaves. Liu et al. (2015) found that there are some regional differences of contents of five main effective components (aucubin, geniposidic acid, chlorogenic acid, catechin and rutin) in EUO leaf. There were no significant differences in HSI, VSI, and CF among all groups in this study, which were similar to the report of crucian carp (Shi et al., 2008) and grass carp (Sun et al., 2017a, b, 2018).

In the present study, no effect of dietary EUO was observed in muscle moisture, crude protein, and crude ash contents of rainbow trout, as reported for $C$. autatus gibelio (Meng et al., 2007), L. vannamei (Liu, 2013), C. idella (Sun et al., 2017a, b). The lipid storage is one of the key factors in determining skeletal muscle quality. In previous studies, Sun et al. (2018) found that dietary EUO could decreased muscle lipid content of $C$. idella. However, the other studies by Sun et al. $(2017 \mathrm{a}, \mathrm{b})$ indicated that there was no significant difference in muscle lipid content of $C$. idella fed EUO-supplemented diet and control diet, which was similar to the studies of L. vannamei (Liu, 2013), C. autatus gibelio (Shi et al., 2008), Angulla japonica (Tanimoto et al., 1993a). At present, the result showed that dietary EUO significantly increased the lipid content in muscle. The result was in line with previous studies in some domestic animals such as pig (Wang et al., 2007), sheep (Yang et al., 2017). It is probably due to the regulation of lipid metabolism and distribution by EUO, which could lower serum lipid levels and increase the muscle crude lipid contents (Yang et al., 2017).

Amino acids are known as anabolic factors, which induce protein gain by stimulating protein synthesis while inhibiting proteolysis (Li et al., 2007; Métayer et al., 2008). Sun et al. (2017a) found that the supplementation of EUO into diet showed significantly improved the threonine (Thr), valine (Val), isoleucine (Ile), leucine (Leu), lysine (Lys), arginine (Arg), proline (Pro), total amino acids (TAA) level in muscle of $C$. idella. The study of Sun et al. (2018) reported that dietary EUO significantly promoted the muscle Arg, aspartic acid (Asp), tyrosine (Tyr), Pro, total nonessential amino acids (TNEAA), delicious amino acids (DAA), TAA levels in C. idella. In the study by Wang et al. (2012), dietary EUO promoted the generation of serine (Ser), glutamic acid (Glu) and lys in 
the muscle of chickens. The present results indicated that phenylalanine (Phe), glycine (Gly), alanine (Ala), DAA in muscle were increased by the supplementation of high levels of EUO. The higher EAA content in muscle means better nutritional value for human. The fresh taste of meat is fundamentally influenced by the content of DAA.Thus it can be seen that dietary EUO can improve the contents of part EAA and DAA in muscle of animals. This may be due to the chlorogenic acid and geniposidic acid, which are the most important bioactive compounds in EU. Sun et al. (2017a) showed that the supplementation of 400$800 \mathrm{mg} / \mathrm{kg}$ chlorogenic acid significantly increased the contents of TEAA and TAA in muscle of grass carp. Zheng et al. (2014) found that dietary chlorogenic acid increased serum Gly level and liver glutathione level of rat. Sun et al. (2018) reported that TNEAA, TAA, DAA in muscle of grass carp were increased by the supplementation of high levels of geniposidic acid. However, we do not know the mechanism of the increased amino acids by EUO or active ingredients of EUO.

Fatty acid composition is an important characteristic of lipid and oil. In this study, 22:1n-9 content in muscle examined are positively correlated to high level of EUO in diet. This is most likely because of EUO contains 22:1n9 (Liu, 2013), which were accumulated in muscle of fish fed EUO diets. Our results showed that 18:3n-6 content in muscle of fish fed EUO diet was lower than that of fish fed control diet, especial for EUO-5 and EUO-40 groups. Similarly, 18:2n-6 content in muscle was decreased with the increasing of EUO level in diets. Contrary to 18:2n6 and 18:3n-6, 20:4n-6 content in muscle of EUO-10 and EUO-40 groups examined were slightly above that of control group. It is possible that rainbow trout has capacity to convert $18: 2 n-6$ to $20: 4 n-6$ (from $18: 2 n-6$ to 18:3n-6, and eventually to 20:4n-6) (Thanuthong et al., 2011). At present, there has been no study on the effect of EUO on muscle fatty acid composition of aquatic animal. Results of this report showed that muscle 22:5n-3 and 22:6n-3 contents were significantly increased by the supplementation of EUO-40 in diet. This may be due to the chlorogenic acid in EUO. Kühn et al. (2017) found that there was a tendency to increase C22:6n-3 levels in the liver of Atlantic salmon fed chlorogenic acid diets. The chlorogenic acid is an ester of caffeic acid and quinic acid present in coffee and various other plants, such as EUO, fruits, vegetables, black teas, soybeans and wheat (Sun et al., 2017b). Chlorogenic acid inclusion seems to affect lipid metabolism. The antioxidant capacity of Chlorogenic acid may delay the oxidation of lipids. And it not only promotes $\beta$-oxidation of lipids but also induces n-3 LC-PUFA syntheses via transcriptional control of fatty acid elongases and desaturases through increased the expression of the perxisome proliferator-acivated receptor a (Rakhshandehroo et al., 2007; Kühn et al., 2017). However, it did not showed dose-dependent between chlorogenic acid addition level and muscle n-3 LC-PUFA level in this study.

In conclusion, the present study demonstrates that the supplementation of EUO could improve flesh quality without negative effects on growth performance of juvenile rainbow trout, Oncorhynchus mykiss. The supplemental level of EUO was estimate to be a $40.00 \mathrm{~g} \mathrm{~kg}^{-1}$ diet.

\section{ACKNOWLEDGEMENT}

This research was supported by grants from Breeding and Reproduction in The Plateau Mountainous Region, Ministry of Education (Guizhou University) (No. GYSD-K-2018-04), the Science and Technology Project of Guizhou Province (No. 20162502, No. 20172532), Guizhou province science and technology platform and talent team plans (No. 20185253), the China Agriculture Research System (No. CARS-46).

\section{Statement of conflict of interest}

The authors declare no conflict of interest.

\section{REFERENCES}

Abedi, E. and Sahari, M.A., 2014. Long-chain polyunsaturated fatty acid sources and evaluation of their nutrition and functional properties. Fd. Sci. Nutr., 2: 443-463. https://doi.org/10.1002/fsn3.121

AOAC, 2005. Official methods of analysis of the association of analytical chemistry, $18^{\text {th }} \mathrm{ed}$, Chapter 39, (Code 39.1.15). Association of Official Analytical Chemists, Washington, DC, pp. 5-8.

Bermejo-Poza, R., Fuente, J.D.L., Pérez, C., Chavarri, E.G.D., Diaz, M.T., Torrent, F. and Villarroel, M., 2017. Determination of optimal degree days of fasting before slaughter in rainbow trout (Oncorhynchus mykiss). Aquaculture, 473: 272-277. https://doi.org/10.1016/j.aquaculture.2017.01.036

Cui, W.D., Leng, X.J., Li, X.Q., Li, X.N. and Xu, J., 2009. Effect of astaxanthin and canthaxanthin pigmentation of muscle and total antioxidant capacity of liver in rainbow trout (Oncorhynchus mykiss). J. Fish. China, 33: 987-995.

Folch, J., Lees, M. and Sloane-Stanley, G., 1957. A simple method for the isolation and purification of total lipids from animal tissues. J. biol. Chem., 226: 497-509.

Geun, W.L., Hyun, C.Y. and Sang, Y,B., 2004. Inhibitory effect of Eucommia ulmoides Oliver on adipogenic differentiation through proteome analysis. Enzyme Microb. Technol., 35: 632-638. https://doi. 
org/10.1016/j.enzmictec.2004.08.037

Grigorakis, K., 2007. Compositional and organoleptic quality of fanned and wild gilthead sea bream (Sparus aurata) and sea bass (Dicentrarchus labrax) and factors affecting it: A review. Aquaculture, 272: 55-75. https://doi.org/10.1016/j. aquaculture.2007.04.062

Jiao, H.L., Liu, Y.L. and Zhang, D.D., 2015. Qualities of Eucommia ulmoides seeds oils from different areas and oil extraction techniques. Cereals and Oils, 28: 53-57.

Kamireddy, N., Jittinandana, S., Kenney, P.B., Slider, S.D., Kiser, R.A., Mazik, P.M. and Hankins, J.A., 2011. Effect of dietary vitamin E supplementation an refrigerated storage on quality of rainbow trout fillets. J. Fd. Sci., 76: S233-S241. https://doi. org/10.1111/j.1750-3841.2011.02121.x

Kühn, G., Pallauf, K., García, J., Segura, J., Ipharraguerre, I., Merue, A., Stubhaug, I., Koppe, W., Rimbach, G. and Menoyo. D., 2017. Effect of diets low in fish oil and supplemented with chlorogenic acid on fatty acid composition and lipid metabolism in Atlantic salmon (Salmo salar L.). Aquacul. Nutr., 23: 730740. https://doi.org/10.1111/anu.12440

Kwon, S.H., Ma, S.X., Hong, S.I., Kim, S.K., Lee, S.Y. and Jang, C.G., 2014. Eucommia ulmoides Oliv. Bark. attenuates 6-hydroxydopamine-induced neuronal cell death through inhibition of oxidative stress. J. Ethnopharmacol., 152: 173-182. https:// doi.org/10.1016/j.jep.2013.12.048

Leng, X.J., Meng, X.L., Li, J.L., Li, X.L. and Hua, X.M., 2008. Effect of Duzhong (Eucommia ulmoides Oliver) leaf on growth, serum non-specific immune index and meat quality of grass carp. J. Fish. China, 32: 434-440.

Li, P., Yin, Y.L., Li, D.F., Kim, S.W. and Wu, G.Y., 2007. Amino acids and immune function. $B r$. J. Nutr., 98: 237-252. https://doi.org/10.1017/ S000711450769936X

Liu, R.H., Tang, F.R., Chen, L.Y., Shao, F., Li, Y.Y., Zhang, Z.L. and Huang, H.L., 2015. Comparison of contents of five effective components in Eucommiae folium from different areas. Chinese $J$. exp. tradit. med. Formulae, 21: 31-34.

Liu, Y.L., 2013. Research on in vitro culture and seed oil composition of Eucommia ulmoides Oliv. Master's Thesis, Shaanxi University of Technology, Hanzhong, China.

Meng, X.L., Leng, X.J., Li, X.Q., Lun, F., Liu, X.M., Li, B.S. and Li, J.L., 2007. Effect of Eucommia ulmoides on growth and serum non-specific immune index of grass carp (Ctenopharyngodon idellus) fingerling. J. Shanghai Fish. Univ., 16: 329-333.

Métayer, S., Seiliez, I., Collin, A., Duchêne, S., Mercier, Y., Geraert, P.A. and Tesseraud, S., 2008. Mechanisms through which sulfur amino acids control protein metabolism and oxidative status. J. nutr. Biochem., 19: 207-215. https://doi. org/10.1016/j.jnutbio.2007.05.006

Mirghaed, A.T., Ghelichpour, M., Zargari, A. and Yousefi, M., 2018. Anaesthetic efficacy and biochemical effects of 1,8-cineole in rainbow trout (Oncorhynchus mykiss, Walbaum, 1792). Aquacul. Res., 49: 2156-2165. https://doi.org/10.1111/ are. 13671

MOAC, 2019. China fisheries yearbook. China Agriculture Publisher, Beijing, China.

Morrison, W.R. and Smith, L.M., 1964. Preparation of fatty acid methyl esters and dimethylacetals from lipids with boron fluoride-methanol. J. Lipid Res., 5: 600-608.

Okada, N., Shirata, K. and Niwano, M., 1994. Immunosuppressive activity of a monoterpene from Eucommia ulmoides. Phytochemistry, 37: 281-282. https://doi.org/10.1016/0031-9422(94)85042-9

Poli, B.M., 2009. Farmed fish welfare-suffering assessment and impact on product quality. Italian J. Anim. Sci., 8(Supp-1): 139-160. https://doi. org/10.4081/ijas.2009.s1.139

Pottinger, T.G., 2001. Effects of husbandry stress on flesh quality indicator in fish. In: Farmed fish quality (eds. S.C. Kestin and P.D. Warriss). Blackwell Sciences, London, UK, pp. 145-160.

Rakhshandehroo, M., Sanderson, L.M., Matilainen, M., Stienstra, R., Carlberg, C., de Groot, P.J., Müller, M. and Kersten, S., 2007. Comprehensive analysis of PPARa-dependent regulation of hepatic lipid metabolism by expression profiling. PPAR Res., 27: 1-13. https://doi.org/10.1155/2007/26839

Rezaei, M. and Hosseini, S.F., 2008. Quality assessment of farmed rainbow trout (Oncorhynchus mykiss) during chilled storage. J. Fd. Sci., 73: 93-96. https:// doi.org/10.1111/j.1750-3841.2008.00792.x

Shearer, K.D., 2001. The effect of diet composition and feeding regime on the proximate composition of farmed fishes. In: Farmed fish quality (eds. S.C. Kestin and P.D. Warriss). Blackwell Sciences, London, UK, pp. 31-41.

Shi, Y., Leng, X.J., Li, X.Q., Li, B.S. and Hu, B., 2008. Effect of Du-zhong (Eucommia ulmoides Oliver) leaf extract on growth, serum non-specific immune response and meat quality of Crucian carp. $J$. Zhejiang Univ., 34: 200-206. 
Si, C.L., Liu, S.C., Xu, G.H., Ren, X.D., Yu, G.J. and $\mathrm{Wu}, \mathrm{L} ., 2$ 2013. Chemical constituents of the root barks of Eucommia ulmoides. Chem. nat. Compounds, 49: 974-976. https://doi.org/10.1007/ s10600-013-0799-2

Suárez, M., García-Gallego, M., Trenzado, C., GuilGuerrero, J., Furne, M., Domezain, A., Alba, I. and Sanz, A., 2014. Influence of dietary lipids and culture density on rainbow trout (Oncorhynchus mykiss) flesh composition and quality parameter. Aquacul. Engin., 63: 16-24. https://doi. org/10.1016/j.aquaeng.2014.09.001

Sun, W.T., Li, X.Q., Xu, H.B., Chen, J.N., Xu, X.Y. and Leng, X.J., 2017a. Effects of dietary chlorogenic acid on growth, flesh quality and serum biochemical indices of grass carp (Ctenopharyngodon idella). Aquacul. Nutr., 23: 1254-1263. https://doi. org/10.1111/anu.12500

Sun, W.T., Li, X.Q., Xu, H.B., Chen, J.N., Xu, X.Y. and Leng, X.J., 2017b. Effects of dietary geniposidic acid on growth, flesh quality and lipid metabolism of grass carp (Ctenopharyngodon idella). J. World Aquacul. Soc., 48: 927-937. https://doi.org/10.1111/ jwas. 12412

Sun, W.T., Xu, X.Y., Li, X.Q., Pan, W.Q. and Leng, X.J., 2018. Effects of dietary geniposidic acid on growth, flesh quality and collagen gene expression of grass carp (Ctenopharyngodon idella). Aquacul. Nutr., 24: 1112-1121. https://doi.org/10.1111/anu.12650

Tanimoto, S.Y., Ikuma, K. and Takahashi, S., 1993a. Improvement in raw meat texture of cultured eel by feeding of tochu leaf powder. Biosci. Biotechnol. Biochem., 57: 205-208. https://doi.org/10.1271/ bbb. 57.205

Tanimoto, S.Y., Koike, K. and Takahashi, S., 1993 b. Improvement in broiled meat texture of cultured ell by feeding of tuchu leaf powder. Biosci. Biotechnol. Biochem., 57: 325-327. https://doi.org/10.1271/ bbb. 57.325

Thanuthong, T., Francis, D.S., Senadheera, S.P.S.D., Jones, P.L. and Turchini, G.M., 2011. LC-PUFA biosynthesis in rainbow trout is substrate limited: Use of the whole body fatty acid balance method and different 18:3n-3/18:2n-6 ratios. Lipids, 46: 1111-1127. https://doi.org/10.1007/s11745-011$3607-4$

Wang, J.H., He, J.H., Yi, X., Li, Y. and Zhao, Y.R., 2007. Effect of Eucommia ulmoides Oliv. extract on growth performance, carcass characteristics, meat qualities and muscular amino acid contents in growing pig. Chinese J. Anim. Nutr., 19: 269-276.

Wang, M.Q., Du, Y.J., Ye, S.S., Tao, W.J. and Wang, C., 2012. Effects of Duzhong (Eucommia ulmoides Oliv.) on growth performance and meat quality in broiler chicks. J. Anim. Vet. Adv., 11: 1385-1389. https://doi.org/10.3923/javaa.2012.1385.1389

Wang, L., Wu, L., Liu, Q., Zhang, D.F., Yin, J.J., Xu, Z. and Zhang, X.Z., 2018. Improvement of flesh quality in rainbow trout (Oncorhynchus mykiss) fed supranutritional dietary selenium yeast is associated with the inhibited muscle protein degradation. Aquacul. Nutr., 24: 1351-1360. https:// doi.org/10.1111/anu.12672

Woynarovich, A., Hoitsy, G. and Moth-Poulsen, T., 2011. Small-scale rainbow trout farming. Retrieved from: http://www.fao.org/docrep/015/i2125e/ i2125e.pdf

Wu, Y.P., Feng, L., Jiang, W.D., Liu, Y., Jiang, J., Li, S.H., Tang, L., Kuang, S.Y. and Zhou, X.Q., 2015. Influence of dietary zinc on muscle composition, flesh quality and muscle antioxidant status of young grass carp (Ctenopharyngodon idella Val.). Aquacul. Res., 46: 2360-2373. https://doi. org/10.1111/are.12392

Yang, G.Q., Wang, L.F., Zhu, H.S., Jia, S.D., Du, Y.H., Cao, Y.L., Zhao, Z.W., Guo, W.J., Hu, C. and Li, M., 2017. Effects of Eucommia ulmoides leaves on sheep lipid metabolism and the mechanism. Chinese J. Anim. Nutr., 29: 3635-3647.

Yildiz, M., Köse, I., Issa, G. and Kahraman, T., 2015. Effect of different plant oil growth performance, fatty acid composition and flesh quality of rainbow trout (Oncorhynchus mykiss). Aquacul. Res., 46: 2885-2896. https://doi.org/10.1111/are.12441

Zheng, R., Yang, Y.H., Zhou, Y., Wen, Y.M., Ding, S., Liu, G., Wu, X., Liao, P., Deng, Z.Y., Assaad, H., Wu, G.Y. and Yin, Y.L., 2014. Metabolomic analysis of amino acid and energy metabolism in rats supplemented with chlorogenic acid. Amino Acids, 46: 2219-2229. https://doi.org/10.1007/ s00726-014-1762-7

Zhou, J.F., Zhang, T.M., Chen, W.A. and Liang, Y.Z., 2009. Comparative analysis of chemical components between barks and leaves of Eucommia ulmoides Oliver. J. Central South Univ., 16: 371379. https://doi.org/10.1007/s11771-009-0063-x 